\title{
Plastoglobules: a new address for targeting recombinant proteins in the chloroplast
} Pierre-Alexandre Vidi1,2, Felix Kessler*1 and Claire Bréhélin ${ }^{1}$

\author{
Address: ${ }^{1}$ Institute of Botany, University of Neuchâtel, rue Emile Argand 11, CP158, CH-2009 Neuchâtel, Switzerland and ${ }^{2}$ Department of \\ Medicinal Chemistry and Molecular Pharmacology, Purdue University, 575 Stadium Mall Dr., RHPH 210, West Lafayette, IN 47907, USA \\ Email: Pierre-Alexandre Vidi - pvidi@purdue.edu; Felix Kessler* - Felix.kessler@unine.ch; Claire Bréhélin - claire.brehelin@unine.ch \\ * Corresponding author
}

Published: 10 January 2007

BMC Biotechnology 2007, 7:4 doi:10.1 186/1472-6750-7-4
Received: 22 September 2006

Accepted: 10 January 2007

This article is available from: http://www.biomedcentral.com/I472-6750/7/4

(c) 2007 Vidi et al; licensee BioMed Central Ltd.

This is an Open Access article distributed under the terms of the Creative Commons Attribution License (http://creativecommons.org/licenses/by/2.0), which permits unrestricted use, distribution, and reproduction in any medium, provided the original work is properly cited.

\begin{abstract}
Background: The potential of transgenic plants for cost-effective production of pharmaceutical molecules is now becoming apparent. Plants have the advantage over established fermentation systems (bacterial, yeast or animal cell cultures) to circumvent the risk of pathogen contamination, to be amenable to large scaling up and to necessitate only established farming procedures. Chloroplasts have proven a useful cellular compartment for protein accumulation owing to their large size and number, as well as the possibility for organellar transformation. They therefore represent the targeting destination of choice for recombinant proteins in leaf crops such as tobacco. Extraction and purification of recombinant proteins from leaf material contribute to a large extent to the production costs. Developing new strategies facilitating these processes is therefore necessary.
\end{abstract}

Results: Here, we evaluated plastoglobule lipoprotein particles as a new subchloroplastic destination for recombinant proteins. The yellow fluorescent protein as a trackable cargo was targeted to plastoglobules when fused to plastoglobulin 34 (PGL34) as the carrier. Similar to adipocyte differentiation related protein (ADRP) in animal cells, most of the protein sequence of PGL34 was necessary for targeting to lipid bodies. The recombinant protein was efficiently enriched in plastoglobules isolated by simple flotation centrifugation. The viability of plants overproducing the recombinant protein was not affected, indicating that plastoglobule targeting did not significantly impair photosynthesis or sugar metabolism.

Conclusion: Our data identify plastoglobules as a new targeting destination for recombinant protein in leaf crops. The wide-spread presence of plastoglobules and plastoglobulins in crop species promises applications comparable to those of transgenic oilbody-oleosin technology in molecular farming.

\section{Background}

Recombinant proteins are massively used in medicine as diagnostic reagents, drugs or vaccines. Moreover, the rapid discovery of new pharmaceutical proteins leads to an increased demand for their production [1]. To date, pro- duction of recombinant proteins mainly relies on microbial fermentation or on insect and mammalian cell cultures. These systems allow highly controlled manufacturing procedures essential for product quality. However, they have disadvantages in term of cost and scalability. 
Pathogen contamination of animal cell cultures also represent an important safety issue.

Plants are now being recognised as an alternative system for the production of recombinant proteins. Plant expression systems allow large scale production of recombinant proteins with accurate folding and assembly of protein complexes [2-5]. Importantly, plant systems offer the possibility of lowering production costs by a factor of 10 to 100 compared to traditional systems $[1,6]$. Chloroplasts have proven a useful cellular compartment for protein accumulation owing to their large size and number. Moreover, transplastomic plants (produced by introducing DNA into the chloroplast genome) enable high yields in recombinant proteins due to a high transgene copy number and limited epigenetic phenomena $[7,8]$. Contamination of wild and crop species by pollen flow is also largely circumvented by organellar transformation [7].

Important issues for industrial production of plantderived recombinant proteins are extraction and purification [9]. Standard protocols include homogenisation of plant biomass followed by chromatographic methods. However, high abundance of secondary compounds, especially in tobacco, is problematic for chromatographic procedures. Therefore, developing a cost-effective preliminary (or alternative) purification step is required [6].

The chloroplast has several interior compartments: the envelope membranes, the thylakoid membranes and lumen, the stroma and plastoglobules. So far, the stroma has been the major target for protein accumulation in plastids [8]. Plastoglobules are low density lipoprotein particles attached to the thylakoid membranes [10] (see
Fig. 1) and coated with proteins of the PAP-fibrillin family [11-13]. The recently determined proteome [14,15] of Arabidopsis plastoglobules identified eight PAP-fibrillin proteins ("plastoglobulins").

In this study, we examined the sequence requirement for correct targeting of the plastoglobulin of $34 \mathrm{kDa}$ (PGL34, [AGI: At3g58010]). We then used the genomic sequence of PGL34 to target a fluorescent protein (YFP) to plastoglobules and took advantage of the low density of plastoglobules for rapid enrichment of the recombinant protein by flotation centrifugation, YFP allowing detection throughout the procedure.

\section{Results \\ Sequence requirement for targeting of PGL34-GFP to plastoglobules}

In the absence of data on the sequence requirement for plastoglobule targeting, we designed a series of C-terminal GFP fusion constructs that removed portions of PGL34 coding sequence (Fig. 2A). PGL34 is a representative member of the Arabidopsis PAP-fibrillin family.

Three domains with somewhat higher hydrophobic scores can be recognised in the Kyte and Doolittle hydropathy plot of PGL34 ([16], Fig. 2A). These domains comprise residues 80-94 (HI), 142-161(H2) and 273-282 (H3). Furthermore, any domain responsible for targeting or anchoring plastoglobulins to plastoglobules may be conserved among the different members of the PAP-fibrillin family. Sequence alignments (not shown) revealed that a central domain in PGL34 (residues 103 to 132, Fig. 2A) is conserved among the PAP-fibrillin family.

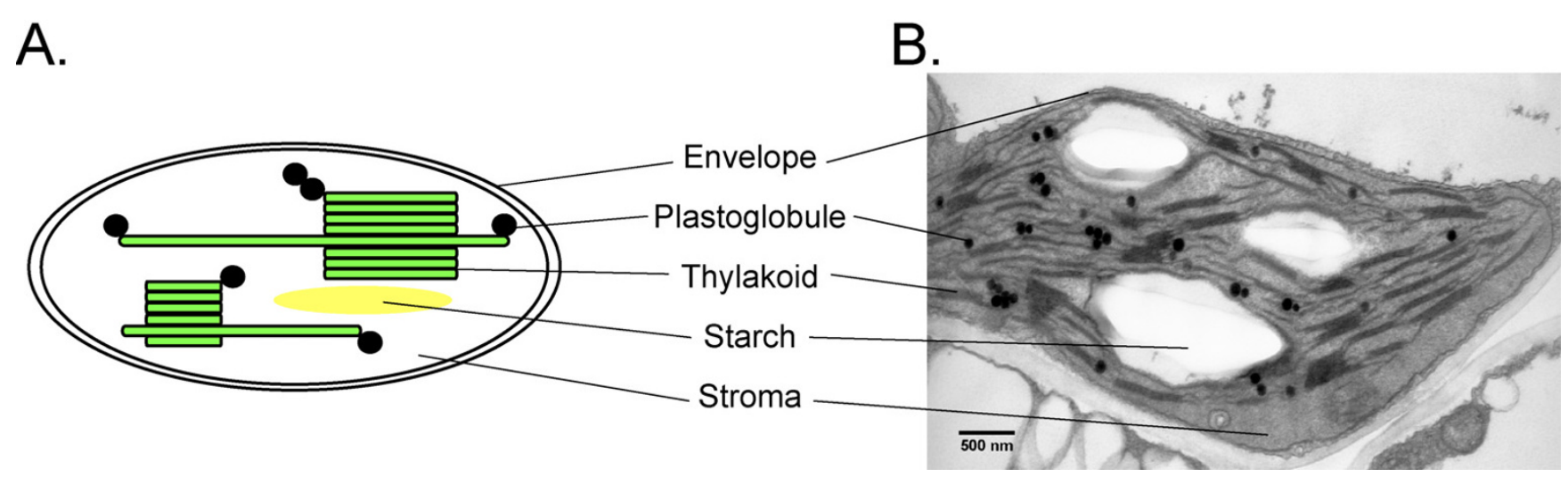

Figure I

Plastoglobule lipoprotein particles in chloroplasts. A. Schematic diagram of a chloroplast. The organelle is delimited by a double membrane system (envelope). The interior of chloroplasts comprises the aqueous stroma, the thylakoid membranes (green) and starch granules (yellow). Plastoglobules (black circles) are lipid particles associated with thylakoids. B. Transmission electron micrograph of an Arabidopsis chloroplast. Plastoglobules are visible as dark round bodies after postfixation with osmium tetroxide. 
A.

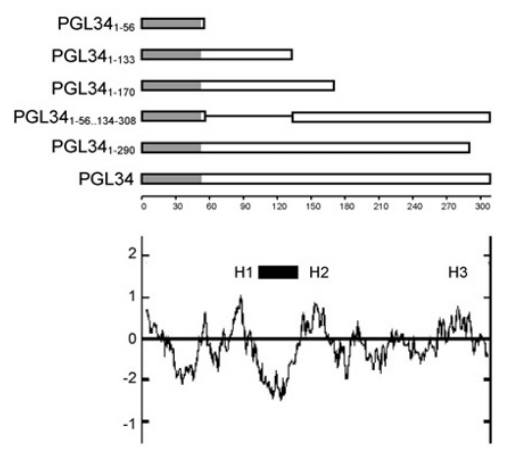

C.

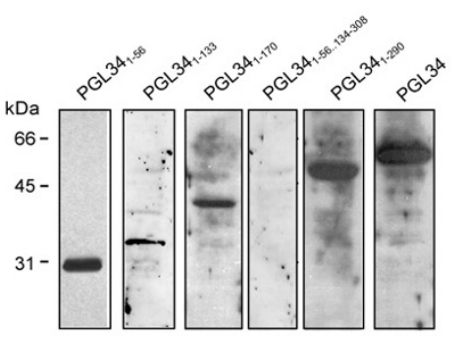

B.

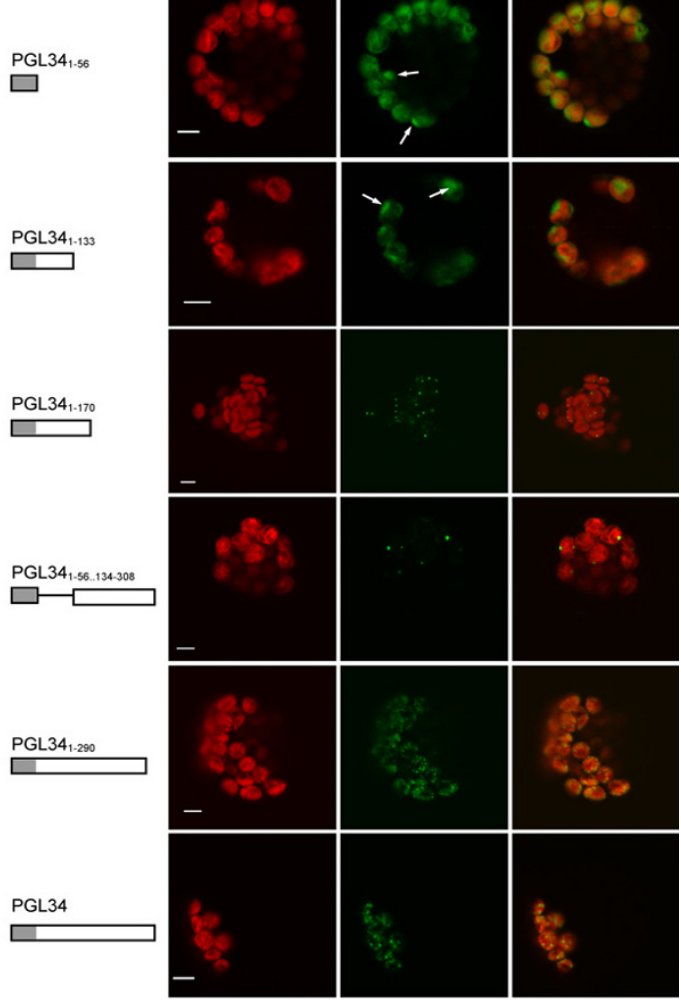

YFP
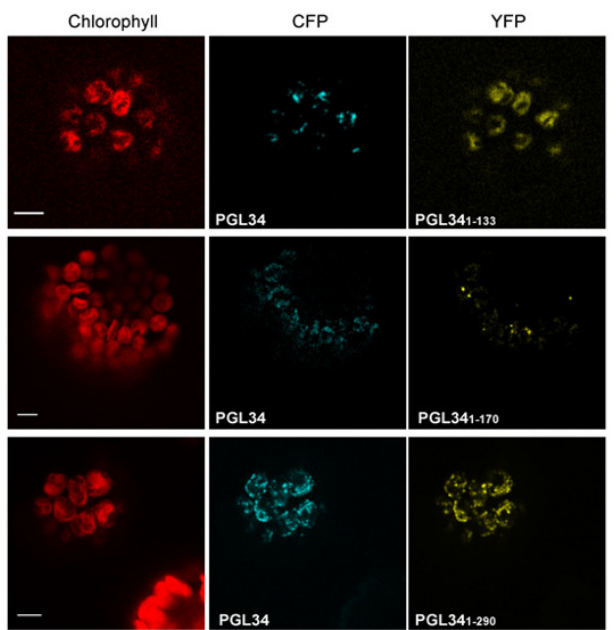

Merge (YFP / CFP)
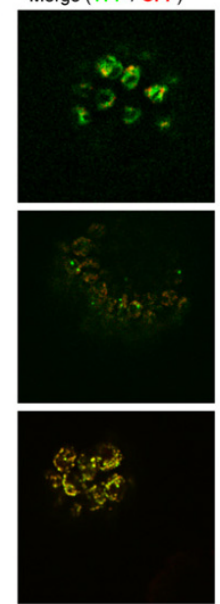

Figure 2

Transient expression of truncated PGL34-GFP fusions in protoplasts. A. DNA constructs encoding fragments of or full length AtPGL34 coding sequence fused to GFP. The transit peptide of PGL34 is shaded. The Kyte and Doolittle hydropathy plot of PGL34 is shown and domains with higher hydropathy scores $(\mathrm{HI}-\mathrm{H} 3)$, as well as a domain conserved among PAP-fibrillin proteins (black bar), are indicated. B. Fluorescence of the GFP fusion proteins (GFP) was detected in transformed protoplasts by confocal laser scanning microscopy. Arrows indicate strong GFP signals overlapping with weak chlorophyll autofluorescence signals (chlorophyll). Merge: overlap of chlorophyll and GFP signals. Scale bars: $5 \mu \mathrm{m}$. C. Detection of GFP fusion proteins in transformed protoplasts by immunoblotting using anti-GFP antibodies. D. Cotransformation of protoplasts with full length and truncated PGL34. Protoplasts coexpressing PGL34-CFP and PGL34 ${ }_{1-133^{-}}$YFP, PGL34 $4_{1-170^{-}}$YFP or PGL34 $_{1-290^{-}}$ YFP were analysed by confocal microscopy. CFP fluorescence (CFP) and YFP fluorescence (YFP) were monitored sequentially using distinct excitation wavelengths and detection windows. Chlorophyll: chlorophyll autofluorescence, merge: superposition of YFP and CFP signals (green and red pseudocolours, respectively). Bar length: $5 \mu \mathrm{m}$. 
Both homology and hydropathic criteria were therefore taken into account for the choice of the deletion constructs. The shortest construct (PGL34 $1-56^{-G F P)}$ only comprised 3 residues in addition to the predicted transit peptide and was designed as a stromal control. The second construct, comprising the amino acids 1-133 (PGL34 $\left.133^{-\mathrm{GFP}}\right)$, contained the $\mathrm{H} 1$ domain as well as the conserved central domain. The construct PGL34 $4_{1-170}$-GFP additionally included the $\mathrm{H} 2$ motif. The fourth construct (PGL34 ${ }_{1-290}$-GFP) comprised H1, H2 and H3 motifs, as well as the conserved domain. An additional N-terminal deletion construct (PGL34 $1-56 . .134-308^{-G F P}$ ), lacking residues $57-133$, contained the $\mathrm{H} 2$ and $\mathrm{H} 3$ motifs but not $\mathrm{H} 1$ and the central domain.

As shown in Fig. 2B, PGL34 ${ }_{1-56}$-GFP and PGL34 $4_{1-133}$-GFP gave diffuse signals broadly overlapping with the autofluorescence of the chlorophyll. In several chloroplasts however, peak GFP and chlorophyll fluorescence did not overlap, suggesting that both fusion proteins localised in the stroma. In contrast, when PGL34 ${ }_{1-290}$-GFP construct was expressed in protoplasts, the fusion protein localised to small punctate structures, similarly to the full length protein. A distinct pattern was observed with PGL34 ${ }_{1-170^{-}}$ GFP and PGL34 $1-56 . .134-308^{-G F P}$ constructs. Fewer and larger fluorescent spots were observed in chloroplasts, suggesting mistargeting or protein aggregation. The same observation was made with constructs comprising residues 1-215 or 1-255 of PGL34 (not shown).

To verify the integrity of each GFP-fusion protein, transformed protoplasts were analysed by Western blotting using an anti-GFP serum (Fig. 2C). With the exception of PGL34 $1-56 . .134-308^{-G F P}$ which could not be detected due to low expression levels, all GFP-fusion proteins migrated at the expected mass. Mistargeting (or aggregation) of PGL34 deletion constructs imply that the corresponding fusion proteins do not colocalise with full-length PGL34. We therefore cotransformed protoplasts with PGL34-CFP and PGL34 deletion constructs fused to YFP (Fig. 2D). As revealed in merged fluorescent images, CFP and YFP signals did not overlap in protoplasts expressing PGL34 and PGL34 $_{1-133}$. In contrast, PGL34-CFP and PGL34 ${ }_{1-290}$-YFP colocalised, indicating that the short C-terminal hydrophilic part of the protein is dispensable for targeting. The strong punctate signals observed in protoplasts expressing PGL34 $4_{1-170}$ were not labelled with PGL34-CFP, confirming mistargeting and/or aggregation of the truncated protein.

\section{Transgenic plants expressing YFP fused at the C-terminus of PGL34}

The results obtained with PGL34-GFP deletion constructs suggested that most of the protein sequence is required for plastoglobule targeting. To address the potential of plas- toglobule targeting for protein purification, Arabidopsis plants were transformed with the coding sequence of YFP replacing the stop codon in the genomic sequence of

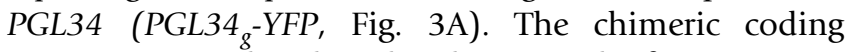
sequence was placed under the control of PGL34 promoter and terminator in order to minimise the risk of gene silencing. Eleven primary transformants $(\mathrm{Tl})$ were isolated and homozygous lines containing a single insertion locus were selected by segregation analysis (not shown). Expression of PGL34 ${ }_{g}$-YFP in these lines was analyzed using both Western blotting and fluorescence microscopy. A wide range of transgene expression levels was observed (Fig. 3B), possibly reflecting positional effects of the T-DNA insertions [17]. A double band was detected in the transgenic plant extracts, the upper band possibly reflecting partial cleavage of the chloroplast targeting sequence.

The T2 line 5.2, accumulating the highest level of recombinant protein, was chosen for subsequent analysis. The concentration of recombinant protein in crude extracts was quantified by comparing immunoblot signals with serial dilutions of purified GFP produced in Escherichia coli (Fig. 3C). PGL34-YFP was calculated to account for approximately $0.2 \%(\mathrm{w} / \mathrm{w})$ of total leaf proteins.

Strongest YFP fluorescence was detected in leaves (Fig. 3D) and roots showed comparably lower YFP signals, consistent with the expression pattern of PGL34 determined by microarray analysis [18]. A detailed picture of PGL34-YFP signals was obtained by confocal microscopy analysis of transgenic leaves (Fig. 3E). Punctate signals were observed in epidermal as well as in mesophyll cells. YFP patterns were similar to those observed in protoplasts transiently expressing PGL34-YFP (Fig. 3B), indicating targeting of the YFP cargo to plastoglobules in planta.

\section{Phenotype of plants accumulating PGL34-YFP}

Transgenic plants accumulating PGL34-YFP were indistinguishable from the wild-type when grown in short or long-day conditions (Fig. 4A). The germination rate of transgenic seeds was equal to wild type (Pearson ChiSquare test, $\mathrm{n}=358, \mathrm{p}=0.33$ ). No difference in fresh weight was observed between wild type and transgenic plants (Fig. 4C), indicating that growth was not altered in plants overexpressing PGL34 $4_{\text {-YFP. }}$

In sub-optimal photosynthetic conditions, the photosystem II (PSII) is subject to photoinhibition, characterised by a decrease of maximum PS II quantum efficiency (Fv/ Fm, [19]). Fluorometry revealed similar Fv/Fm fluorescence values in transgenic vs. wild type leaves (Fig. 4B), ruling out major inhibitory effects of plastoglobule targeting on photosynthesis. 
A.

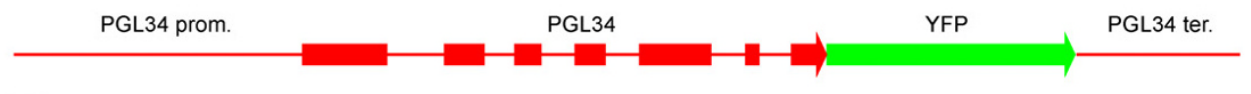

$\overline{0.1 \mathrm{~kb}}$

B.

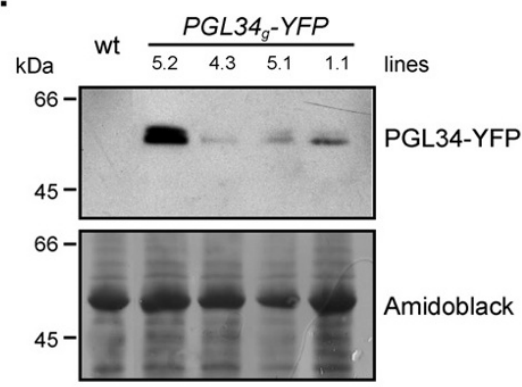

C.

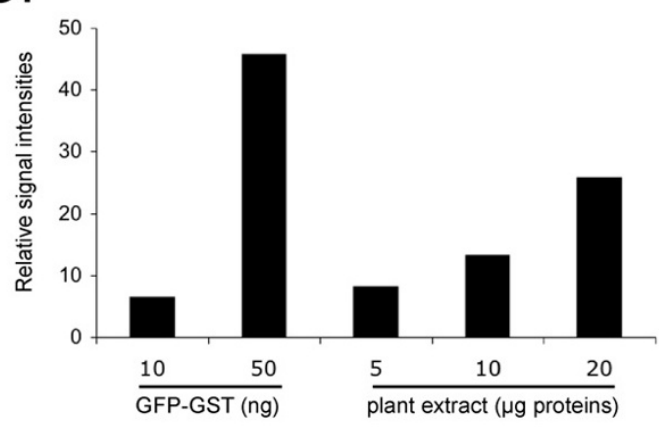

D.

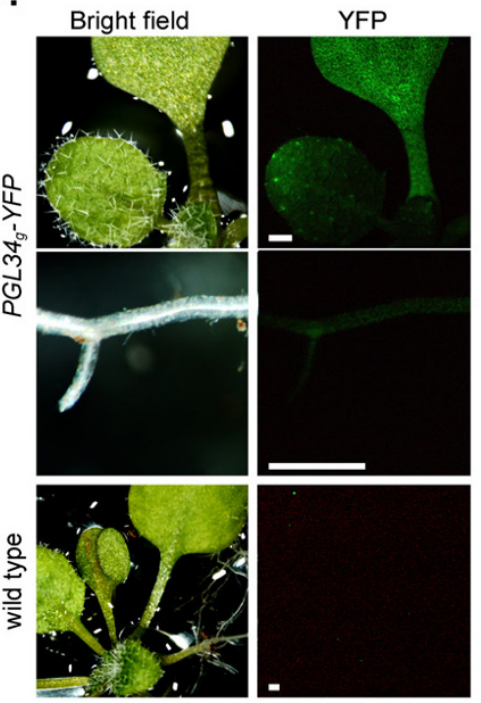

E.
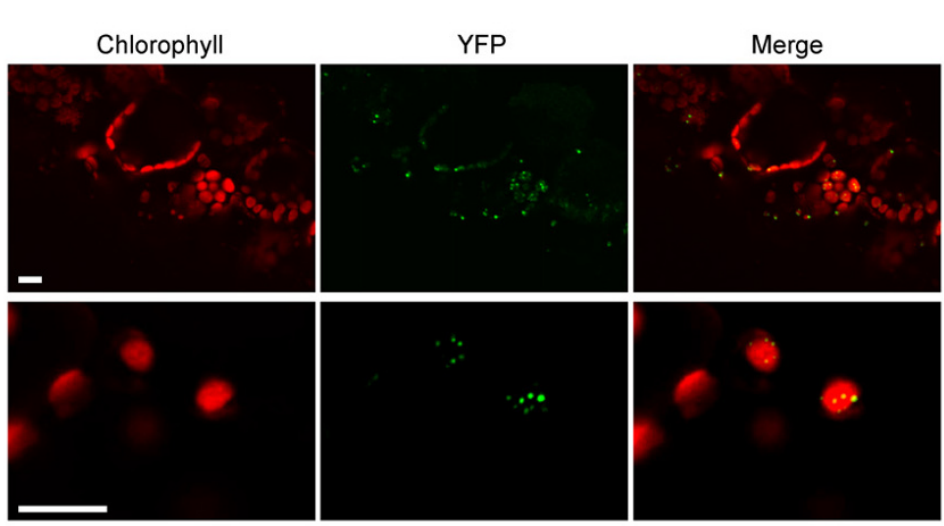

Figure 3

Expression of PGL34 $\mathrm{g}$-YFP in planta. A. Schematic representation of the construct used for plant transformation. The coding sequence of the yellow fluorescent protein (YFP) was inserted in the genomic sequence of PGL34, replacing the PGL34 stop codon. Boxes indicate coding regions. B. Western blot analysis of rosette leaves from homozygous T2 transgenic lines. PGL34-YFP was detected with anti-GFP antibodies. Proteins were visualized on the membrane by staining with amidoblack. $\mathrm{Wt}$, wild type Arabidopsis plant protein extract. C. Yield estimation. Different amounts of total protein extracts from line 5.2 were subjected to SDS-PAGE in parallel with defined amounts of recombinant GST-GFP and chemiluminescent immunoblot signals were quantified. D. Detection of PGL34-YFP by fluorescence microscopy. YFP fluorescence was detected in leaves, cotyledons and roots by illuminating the seedlings with UV light. Scale bars: $0.5 \mathrm{~mm}$. E. Subcellular localization of PGL34-YFP in leaves was determined using confocal laser scanning microscopy. Scale bars: $10 \mu \mathrm{m}$. 
A.

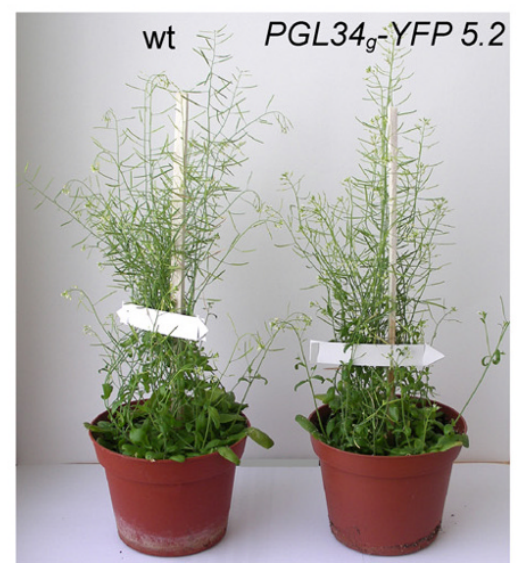

B.

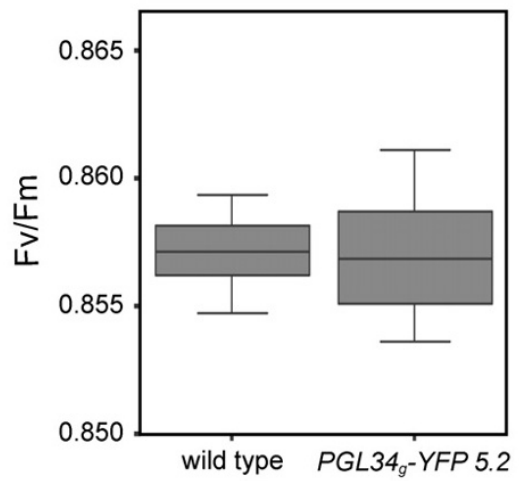

C.

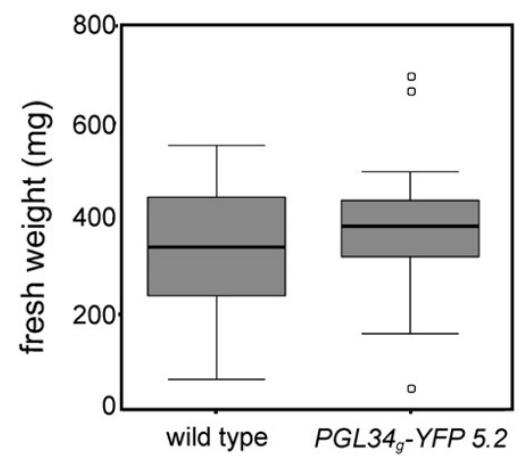

Figure 4

Phenotype of plants expressing PGL34 -YFP. A. Photographs of wild type (wt) or transgenic (line 5.2) plants grown for 8 weeks in $16 / 8$ hours light/dark conditions. B. Maximum photosystem II quantum efficiencies ( $\mathrm{Fv} / \mathrm{Fm}$ ) measured from rosette leaves. T-test, $t=0.17, p=0.67, n=14$. $C$. Fresh weight measured 5 weeks after germination. $T$-test, $t=-1.22, p=0.23$. At least 19 plants were measured. Normal distribution of the data (in B. and C.) was verified using the Kolmogorov-Smirnov test $(p>0.1)$.

\section{Recombinant protein purification by flotation centrifugation}

Plastoglobules were isolated from chloroplast membranes prepared from transgenic leaves using sucrose density gradient flotation centrifugation (see Fig. 5A). Fractions collected from the density gradient were analyzed by SDSPAGE followed by Western blotting (Fig. 5B). The blots were probed with anti-PGL35 (At4g04020), anti-TOC75 (At3g46740) and anti-CAB (chlorophyll a/b binding protein) antibodies as plastoglobule, envelope and thylakoid markers, respectively. The results demonstrated the separation of plastoglobules (fractions 1-7) from envelope (in fractions 11-15) and thylakoid membranes (predominantly in fractions 11-21). PGL34-YFP, detected by antiGFP antibodies, co-distributed with PGL35. Moreover, globular fluorescent structures were highly abundant in low-density fractions (Fig. 5C), demonstrating targeting of the recombinant protein to plastoglobules.

SDS-PAGE analysis of the low-density fractions revealed a prominent band with an apparent mass of $55 \mathrm{kDa}$ consistent with the predicted mass of PGL34-YFP (28.3, processed PGL34 + 26.9, YFP). This band was recognized by anti-GFP antibodies (Fig 5B). Similar to immunodetection on total leaf extracts (Fig. 3a), an additional faint band, migrating at $57 \mathrm{kDa}$, was also recognized by the antibody. Analysis by tandem mass spectrometry confirmed that both bands contained PGL34-YFP (not shown). The upper band may reflect partial cleavage of the chloroplast targeting sequence or post translational modifications of PGL34-YFP. However, mass spectromet- ric analysis of tryptic peptides derived from the two protein bands yielded no additional evidence with regard to the two possibilities.

To estimate the enrichment factor resulting from the gradient flotation, the amount of recombinant protein in the $5 \%$ sucrose step (fraction nb. 6 of the gradient) was determined by comparing immunoblot signals with a dilution series of purified GFP (not shown). PGL34-YFP accounted for about $50 \%$ of the protein content. Based on this and the PGL34-YFP concentration in leaves $(0.2 \% \mathrm{w} / \mathrm{w})$, the gradient flotation step had lead to an approximately 250 fold enrichment of the recombinant protein.

\section{PGL34 homologues in cultivated plant species}

To determine whether proteins similar to PGL34 are present in cultivated plant species, sequence homology searches were performed. As shown in Fig. 6, protein sequences with significant BLAST hits were found in monocots and in a broad range of dicot taxa. Pairwise comparisons performed with the MatGAT software [20] revealed that the protein sequences shown in Fig. 6 were at least $40 \%$ similar. Arabidopsis PGL34 shared for example $79 \%$ similarity and $63 \%$ identity with the protein sequence deduced from a tobacco EST assembly. These data indicate that proteins from the PAP/fibrillin family are highly conserved in the plant kingdom. 


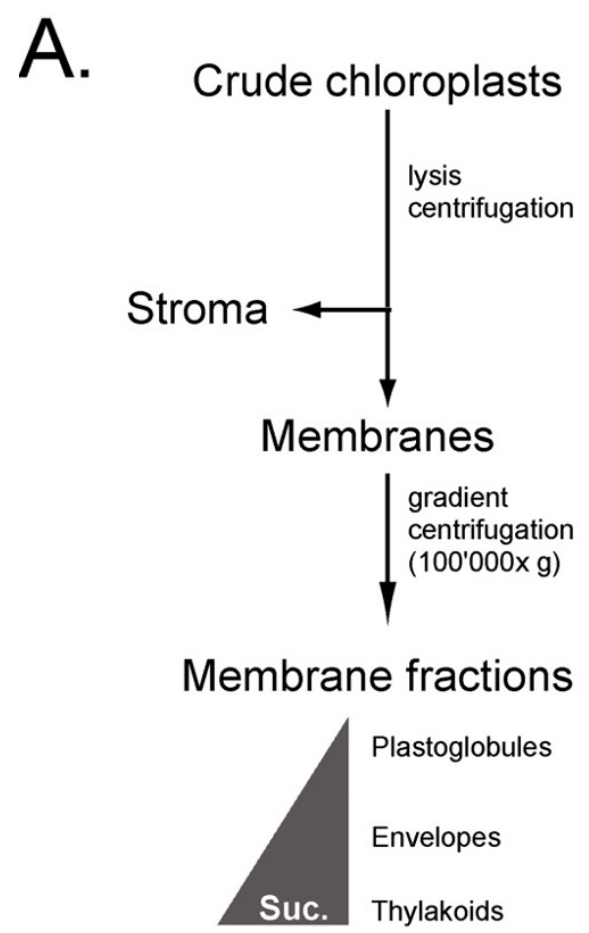

Gradient fraction

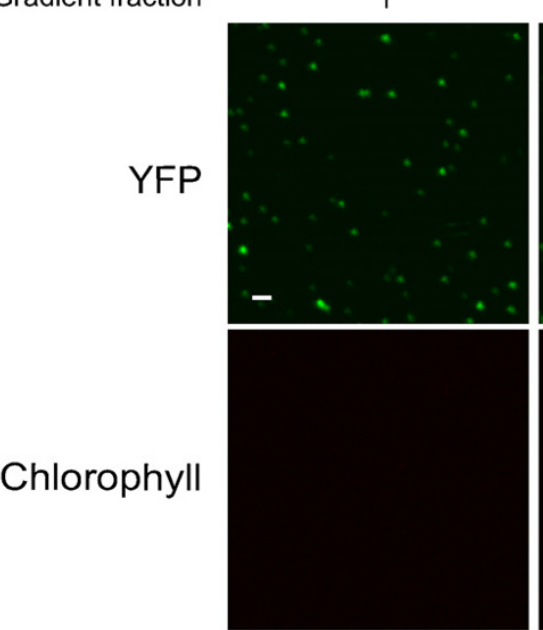

B.

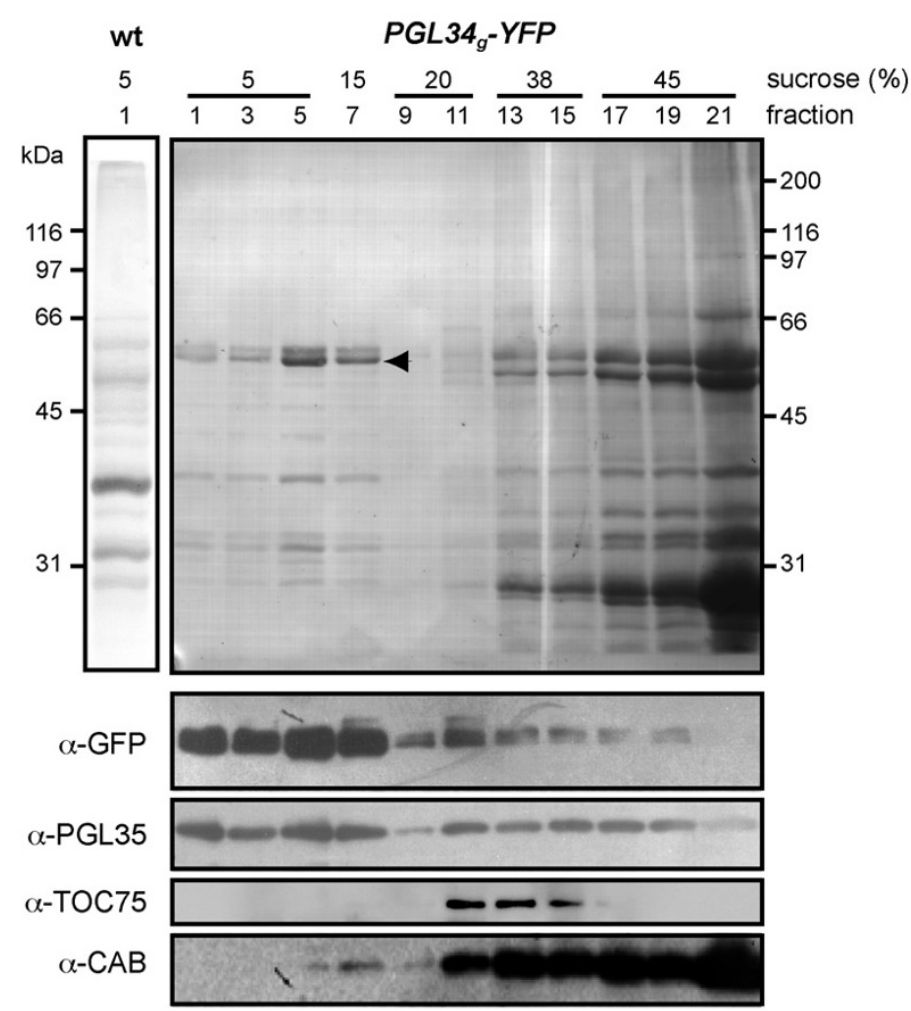

7
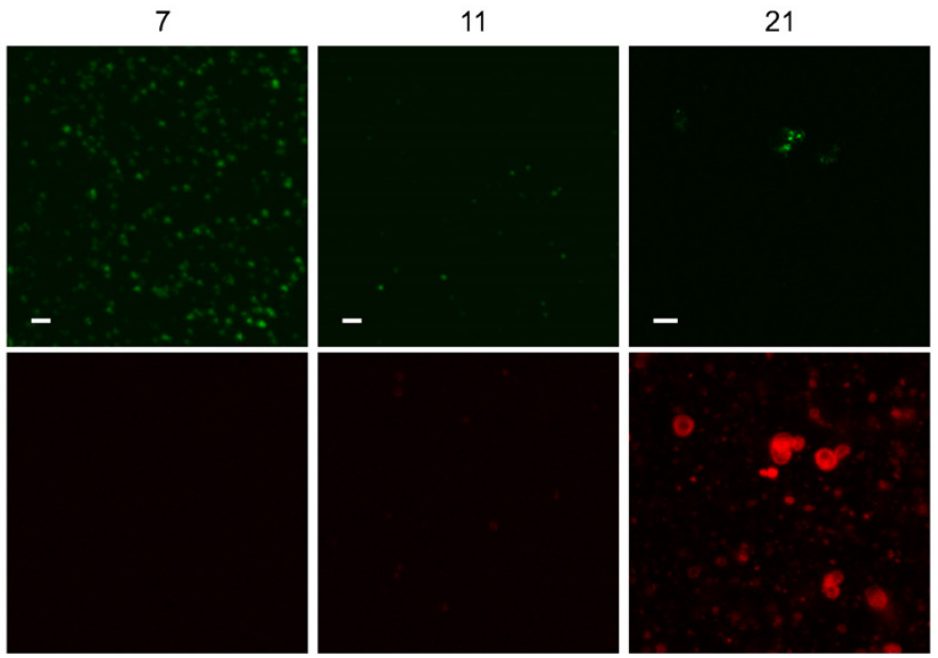

\section{Figure 5}

Purification of PGL34-YFP by flotation centrifugation. A. Diagram illustrating the purification procedure. Suc., sucrose concentration. B. Western blot analysis of fractions from the density gradient. After ultracentrifugation, $0.5 \mathrm{ml}$ fractions were collected starting from the top of the gradient. Proteins contained in $400 \mu \mathrm{l}$ of fractions I-7, $200 \mu \mathrm{l}$ of fractions $9-15,100 \mu \mathrm{l}$ of fractions 17-19 or $50 \mu \mathrm{l}$ of fraction 21 were separated by SDS-PAGE, transferred to a nitrocellulose membrane and stained with amidoblack (upper panel). Silver stained plastoglobule proteins prepared from wild type plants (wt) are shown. Antibodies against GFP, PGL35, TOC75 and CAB were used for immuno blotting, as indicated (lower panels). C. YFP and chlorophyll fluorescence in fractions I, 7, II and 21 was monitored by confocal microscopy. Scale bars: I $\mu \mathrm{m}$ (fractions I-II) or $5 \mu \mathrm{m}$ (fraction 21). 


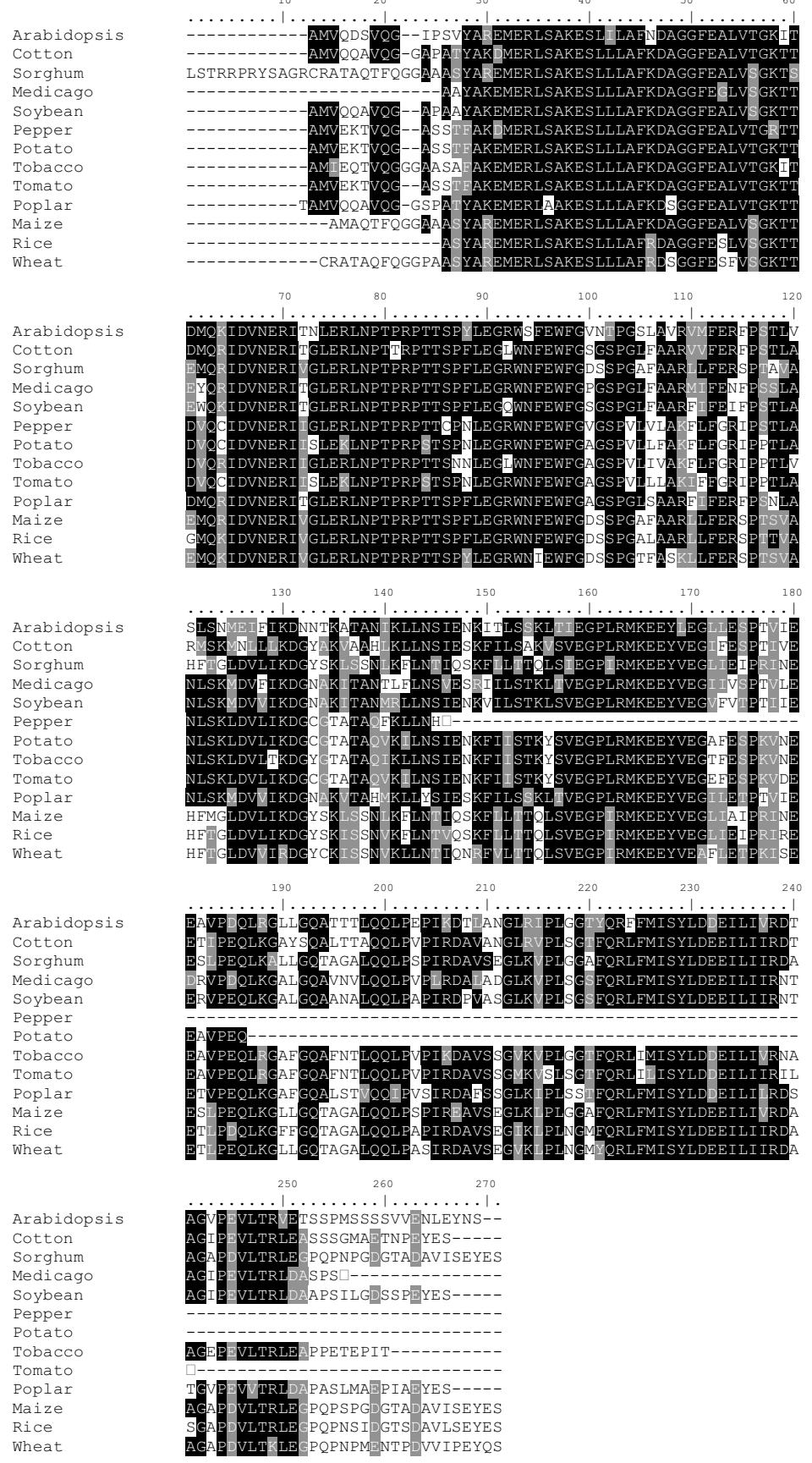

Figure 6

Presence of PGL34 homologues in crop species. Sequence alignment of proteins similar to PGL34. Amino acid sequences were aligned using ClustalW. Transit peptides were predicted using the TargetP software and removed from the protein sequences. The predicted transit peptide length of PGL34 was 53 amino acids. The alignment was formatted with the BOXSHADE 3.2I software. Residues identical in at least 6 sequences are shaded. Identical residues are in black and conserved substitutions are in grey. Diamonds indicate truncated sequences. EST contigs were retrieved from TIGR Plant Transcript Assemblies (TA, http://plantta.tigr.org/search.shtml) and Tentative Consensus (TC, http://tigrblast.tigr.org/tgi/) databases. Cotton, TC6 1795; Sorghum, TC 103242; Medicago, TC 10824I; Soybean, TC228644; Pepper, TC4347; Potato, TC I 20970; Tobacco, TA7467_4097; Tomato, TCI64782; Poplar, TC38I24; Maize, TC299788; Rice, AAO72625 (GenPept); Wheat, TC239733. 


\section{Discussion \\ Sequence requirement for protein targeting to plastoglobules}

The presence of proteins associated with plastoglobules implies that mechanisms ensuring protein assembly in plastoglobules must exist in plastids. However, nothing is known regarding these mechanisms and plastoglobulins do not share conserved sequence motifs with other plant or animal lipid body proteins. Several types of proteins associated with lipid bodies have been described in prokaryotic and eukaryotic cells (reviewed in ref. [21]). They have highly diverse physicochemical properties and topologies, reflecting various modes of association with the lipidic structures. In desiccation tolerant seeds, oil bodies are coated with oleosins [22]. A 72-residue central hydrophobic domain in the proteins, often referred to as "proline knot motif" is essential for their association with oil bodies [23-25]. In mammalian cells, under certain conditions, caveolins accumulate at the surface of cytoplasmic lipid droplets. Using deletion constructs, Ostermeyer et al. [26] demonstrated requirement of a hydrophobic domain for lipid droplet targeting of caveolin-1. Hydrophobic domains were also shown to play important roles in targeting and anchoring perilipin to lipid droplets in adipocytes [27]. If several lipid body proteins are characterised by hydrophobic domains, others lack large apolar regions. Adipophilin (also termed Adipocyte Differentiation-related Protein, ADRP), which localises to the periphery of cytosolic lipid bodies in mammalian cells, has no obvious lipid-binding motif (hydrophobic domains or amphiphatic $\alpha$-helices; [21]) and discontinuous stretches of the protein are necessary for targeting to lipid bodies [28].

Although plastoglobulins associate with lipid bodies, their overall amino acid composition is not hydrophobic (Grand average of hydropathicity (GRAVY) index = -0.173 for PGL34) and it is apparent from hydropathy plots that the proteins, in contrast to oleosins, lack a strongly hydrophobic domain (see Fig. 3A). Therefore, association of PGLs with plastoglobules may rather rely on interactions with surface lipids, as proposed by Kim et al. [29], similarly to ADRP [21]. Based on our observation that the almost complete sequence of PGL34 is necessary to maintain proper targeting (Fig. 2), we propose that a correct folding of PAP/fibrillins rather than a sequence determinant is requested for assembly in plastoglobules.

\section{Similarities between plastoglobules and seed oil-bodies}

A successful technology for production and recovery of recombinant proteins expressed in seeds from oilseed plants has recently been developed at SemBioSys [30,31]. In the so-called Stratosome ${ }^{\mathrm{TM}}$ system, proteins of interest fused to an oleosin moiety are targeted to oilbodies and recovered by subsequent centrifugation steps [32]. Biolog- ically active human insulin has notably been produced from Arabidopsis seeds using this system [33].

Plastoglobules and seed oilbodies share similarities. They are of low density due to their high lipid content and only contain a few different proteins $[14,15,34]$ - both properties being advantageous for purification by density partitioning. Whereas oleosin-coated lipid bodies accumulate predominantly in the cell cytoplasm of oleaginous seeds, in tapetum cells and in pollen grains [22], plastoglobules are ubiquitously found in all types of plastids [35], notably in leaf chloroplasts. In leaf crops such as tobacco producing high biomass, plastoglobule targeting would allow downstream processing of leaf material similar to the oilbody-oleosin system in seeds.

\section{Future perspectives}

In this proof-of-concept study, a strong enrichment in PGL34-YFP was achieved. We expect that the yield of plastoglobulin-fusion proteins will be readily improved in follow-up studies using tissues accumulating plastoglobules or related fibrills such as fruits or senescing leaves [36-38] and/or using plastid genetic engineering [7]. The general usefulness of the plastoglobule-plastoglobulin system is predicted since plastoglobulins as well as plastoglobules are highly conserved throughout the plant kingdom and many homologues have been identified in genome and EST sequencing projects (Fig. 6). In particular leafy crop plants such as tobacco may be ideal to exploit the potential of the plastoglobule targeting system in molecular farming approaches.

\section{Conclusion}

The data presented in this paper demonstrate the potential of protein accumulation in plastoglobules. Sequestration of recombinant proteins in the particles may limit deleterious effects on photosynthetic light and dark reactions. Plastoglobule accumulation combines the advantages offered by a chloroplast localization and, as in seed oil-bodies, of a simple enrichment step needed prior to standard chromatographic purification.

\section{Methods \\ DNA vectors for plant transformation}

The complete coding sequence of PGL34 (At3g58010), excluding the stop codon, was amplified by PCR from a cDNA clones (U15686) obtained from the Arabidopsis Biological Resource Center (ABRC, [39]). Forward (5'-cat gcc ATG GCA TTG ATC CAA CAT GG-3') and reverse (5'cat gcc atg gcA CTG TTG TAT TCA AGA TTC TCT ACA AC3') primers included NcoI sites. The PCR product was ligated in the NcoI site of either pCL60 [40] or pCL62 [14], resulting in C-terminal GFP or CFP fusions respectively, under the control of the CaMV 35S promoter and the nos terminator. 
Partial PGL34 sequences were amplified from pCL60PGL34 using a forward primer containing a XbaI site (5'gct cta gaA TGG CAT TGA TCC AAC ATG G-3') and the following reverse primers, including NcoI sites: 5'-cat gcc atg gcA ACC ATA GCT CTG CAT ATC ATT C-3' (PGL34 ${ }_{1-56}$ ), 5'-cat gcc atg gcA CTC CAT CTA CCT TCA AGA TAA GG-3' (PGL34 $\left.{ }_{1-133}\right), 5^{\prime}$-cat gcc atg gcA TCT TTA ATG AAT ATT TCC A-3' (PGL34 1 170), 5'-cat gcc atg gcT TCC ACC CTC GTT AGA AC-3' (PGL34 1-290). The corresponding PCR products were ligated into the $\mathrm{XbaI}$ and NcoI sites of pCL60 or pCL61 [14], yielding GFP and YFP fusions, respectively. To obtain the deletion construct pCL60PGL34 1-56..134-308, a C-terminal fragment of PGL34 was amplified from pCL60-PGL34 using forward (5'-cat gcc atg gcc TTT GAG TGG TTT GGA GTC AAC-3') and reverse (5'-cat gcc atg gcA CTG TTG TAT TCA AGA TTC TCT ACA AC-3') primers including NcoI sites and ligated in the NcoI site of pCL60-PGL34 $4_{1-56}$. For stable plant transformation, a genomic DNA fragment, including PGL34 coding sequence as well as a $0.85 \mathrm{~kb} 5^{\prime}$ upstream region was amplified by PCR using forward 5'-CAT GCC ATG GAG ATC TTC GGT GAG GAA CAA GAG TT-3' and reverse 5'CAT GCC ATG GCA CTG TTG TAT TCA AGA TTC TCT ACA AC-3' primers and inserted in the NcoI site of the pCL61 vector, in frame with the coding sequence of YFP. PGL34 3' downstream region $(0.47 \mathrm{~kb}$, including the terminator) was amplified by PCR from genomic DNA using forward 5'-CCG CGG CCG CAA ACA GGT TCT CTT GTT ACT CTG ATT C-3' and reverse 5'-GGG CGG CCG GAG ATC TCG GTC TCT CAA AGG ATG TG-3' primers. The fragment was cloned in the NotI site of pCL61. PGL34 and YFP sequences were excised from pCL61 by restriction digest with AatII and KpnI. Fragments were blunted and ligated in the SmaI site of pCAMBIA3300 binary vector (CAMBIA), yielding pCAMBIA3300:PGL34-YFP.

\section{Transient and stable A. thaliana transformation}

Transient transformation of protoplasts was done using the polyethylene glycol method as described in ref. [41], but reducing cellulase and macerozyme (Serva) concentrations to $1 \%$ and $0.25 \%(\mathrm{w} / \mathrm{v})$ respectively. Fluorescence in transformed protoplasts was monitored $48 \mathrm{~h}$ after transformation by confocal laser scanning microscopy. GFP was detected using the FITC (488 nm) laser line from a LEICA TCS 4D microscope (LEICA Microsystems). For double fluorescent experiments, CFP and YFP were detected sequentially with a LEICA SP2 AOBS microscope, using 458 and $514 \mathrm{~nm}$ laser lines and 460-510 $\mathrm{nm}$ and 520-588 nm detection windows, respectively. Chlorophyll autofluorescence was monitored using either 594 nm or TRITC (568 nm) excitation wavelengths.

Stable transformation of $A$. thaliana plants with pCAMBIA3300:PGL34-YFP was carried out using the flo- ral dip method as described [42,43]. Transformants were selected on plates containing phosphinothricin.

\section{Protein extraction and immunoblot analysis}

Proteins were isolated from Arabidopsis leaves according to ref. [44]. $25 \mu \mathrm{g}$ proteins were concentrated by chloroform - methanol precipitation [45], separated by SDSPAGE and blotted onto nitrocellulose membranes. Proteins were stained with amidoblack (= naphthol blue black) for protein detection or used for immunodetection. Blots were probed with anti-GFP (gift from Dr. E. Schäfer) and anti-CAB (kindly provided by Dr. K. Apel) sera, or with affinity-purified antibodies specific for TOC75 [46] and PGL35 [14]. For quantification, chemiluminescent immunoblot signals were analysed using a Bio-Rad ChemiDoc XRS system.

\section{Gradient flotation centrifugation of chloroplast membranes}

Leaves from Arabidopsis plants grown on soil for 4 weeks were harvested and immersed in tap water in the dark at $4^{\circ} \mathrm{C}$ for $30 \mathrm{~min}$. Leaf material was homogenized in $\mathrm{HB}$ buffer (450 mM sorbitol, $20 \mathrm{mM}$ Tricine/KOH pH 8.4, 10 $\mathrm{mM}$ EDTA, $10 \mathrm{mM} \mathrm{NaHCO}{ }_{3}, 1 \mathrm{mM} \mathrm{MnCl}_{2}$ ) with a Waring blender and filtered through cheese cloth and miracloth. Chloroplasts were sedimented $(2 \mathrm{~min} 700 \times \mathrm{g})$, washed with TrE (50 mM Tricine/ $\mathrm{HCl} \mathrm{pH} 7.5,2$ mM EDTA) and hypertonically lysed $10 \mathrm{~min}$ in $\operatorname{TrE}+0.6 \mathrm{M}$ sucrose supplemented with $0.5 \%(\mathrm{v} / \mathrm{v})$ protease inhibitor cocktail (Sigma P9599). The lysate was frozen at $-80^{\circ} \mathrm{C}$, thawed on ice, diluted 3 times with TrE buffer and homogenised with a Potter homogeniser. Total membranes, corresponding to $10 \mathrm{mg}$ of chlorophyll, were sedimented at $100 \mathrm{\prime} 000 \times \mathrm{g}$ and resuspended in $3 \mathrm{~mL} 45 \%$ sucrose in TrE buffer. Membranes were overlaid with a discontinuous sucrose

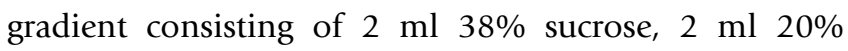

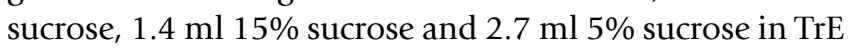
buffer and centrifuged for $17 \mathrm{~h}$ at $100^{\prime} 000 \times \mathrm{g}$ and $4^{\circ} \mathrm{C}$ (SW41Ti rotor, Beckman). $0.5 \mathrm{ml}$ fractions were collected starting from the top of the gradient and used for confocal microscopy analysis or Western blotting (see above).

\section{Fluorometry}

Maximum quantum efficiency of photosystem II (Fv/Fm) was measured using a Handy Plant Efficiency Analyser chlorophyll fluorometer (Hansatech Instruments, Norfolk, UK). Detached leaves from 4 week-old plants were dark-adapted $20 \mathrm{~min}$ prior to measurements.

\section{Authors' contributions}

PV carried out most of the cloning work, transient and stable plant transformation, as well as the microscopic and biochemical analyses and helped to draft the manuscript. CB participated in the elaboration of the experimental design and did the sequence analysis. FK conceived the 
study, drafted the manuscript and was responsible for the research program. All authors read and approved the final manuscript.

\section{Acknowledgements}

We thank Dr. Sacha Baginsky for his expert help with mass spectrometry analysis, Dr. Gabor Csúcs for invaluable help with confocal microscopy, Valère Hofstetter and Christelle Stauffer for technical assistance and Dr. Birgit Agne, Dr. Pia Stieger and Gwendoline Rahim for helpful suggestions on the manuscript. We thank ABRC for providing the cDNA clone. This work was supported by the University of Neuchâtel and in part by the NCCR "Plant Survival" (National Center of Competence in Research).

\section{References}

I. Joshi L, Lopez LC: Bioprospecting in plants for engineered proteins. Curr Opin Plant Biol 2005, 8(2):223-6.

2. Ma JK, Drake PM, Christou P: The production of recombinant pharmaceutical proteins in plants. Nat Rev Genet 2003, 4(10):794-805.

3. Fischer R, Stoger E, Schillberg S, Christou P, Twyman RM: Plantbased production of biopharmaceuticals. Curr Opin Plant Biol 2004, 7(2):152-8.

4. Ma JK, Barros E, Bock R, Christou P, Dale PJ, Dix PJ, Fischer R, Irwin J. Mahoney R, Pezzotti M, Schillberg S, Sparrow P, Stoger E, Twyman RM: Molecular farming for new drugs and vaccines. Current perspectives on the production of Pharmaceuticals in transgenic plants. EMBO Rep 2005, 6(7):593-9.

5. Ma JK, Chikwamba R, Sparrow P, Fischer R, Mahoney R, Twyman RM: Plant-derived Pharmaceuticals - the road forward. Trends Plant Sci 2005, I O(I 2):580-5.

6. Menkhaus TJ, Bai Y, Zhang C, Nikolov ZL, Glatz CE: Considerations for the Recovery of Recombinant Proteins from Plants. Biotechnol Prog 2004, 20:1001-1014.

7. Bock $R$ : Transgenic plastids in basic research and plant biotechnology. I Mol Biol 200I, 3 I 2(3):425-38.

8. Daniell H, Chebolu S, Kumar S, Singleton M, Falconer R: Chloroplast-derived vaccine antigens and other therapeutic proteins. Vaccine 2005, 23(15): I779-83.

9. Giddings G, Allison G, Brooks D, Carter A: Transgenic plants as factories for biopharmaceuticals. Nat Biotechnol 2000, I8(II): II5I-5.

10. Austin JR, Frost E, Vidi PA, Kessler F, Staehelin LA: Plastoglobules are lipoprotein sub-compartments of the chloroplast that are permanently coupled to thylakoid membranes and contain biosynthetic enzymes. Plant Cell 2006, 18:1693-I703.

II. Deruère J, Römer $S$, d'Harlingue A, Backhaus RA, Kuntz M, Camara B: Fibril assembly and carotenoid overaccumulation in chromoplasts: a model for supramolecular lipoprotein structures. Plant Cell 1994, 6: I 19-33.

12. Pozueta-Romero J, Rafia F, Houlne G, Cheniclet C, Carde JP, Schantz ML, Schantz R: A ubiquitous plant housekeeping gene, PAP, encodes a major protein component of bell pepper chromoplasts. Plant Physiol 1997, I I 5(3): I I85-94.

13. Kessler F, Schnell D, Blobel G: Identification of proteins associated with plastoglobules isolated from pea (Pisum sativum L.) chloroplasts. Planta 1999, 208:107-13.

14. Vidi PA, Kanwischer M, Baginsky S, Austin JR, Csucs G, Dörmann P, Kessler F, Brehelin C: Tocopherol cyclase (VTEI) localization and vitamin $E$ accumulation in chloroplast plastoglobule lipoprotein particles. J Biol Chem 2006, 28 I( I6): I | 225-34.

15. Ytterberg AJ, Peltier JB, van Wijk KJ: Protein profiling of plastoglobules in chloroplasts and chromoplasts. A surprising site for differential accumulation of metabolic enzymes. Plant Physiol 2006, 140(3):984-97.

16. Kyte J, Doolittle R: A simple method for displaying the hydropathic character of a protein. J Mol Biol 1982, I 57: I 05- I32.

17. Goodrich J, Tweedie S: Remembrance of things past: chromatin remodeling in plant development. Annu Rev Cell Dev Biol 2002, 18:707-46.

18. Zimmermann P, Hirsch-Hoffmann M, Hennig L, Gruissem W: GENEVESTIGATOR. Arabidopsis microarray database and analysis toolbox. Plant Physiol 2004, 136:2621-32.
19. Bailey S, Thompson E, Nixon PJ, Horton P, Mullineaux CW, Robinson $\mathrm{C}$, Mann $\mathrm{NH}$ : A critical role for the Var2 FtsH homologue of Arabidopsis thaliana in the photosystem II repair cycle in vivo. J Biol Chem 2002, 277(3):2006-I I.

20. Campanella JJ, Bitincka L, Smalley J: MatGAT: an application that generates similarity/identity matrices using protein or DNA sequences. BMC Bioinformatics 2003, 4:29.

21. Murphy DJ: The biogenesis and functions of lipid bodies in animals, plants and microorganisms. Prog Lipid Res 200I, 40(5):325-438.

22. Hsieh K, Huang AH: Endoplasmic reticulum, oleosins, and oils in seeds and tapetum cells. Plant Physiol 2004, I36(3):3427-34.

23. Abell BM, Holbrook LA, Abenes M, Murphy DJ, Hills MJ, Moloney $M M$ : Role of the proline knot motif in oleosin endoplasmic reticulum topology and oil body targeting. Plant Cell 1997, 9(8): $|48|-93$

24. Abell BM, High S, Moloney MM: Membrane protein topology of oleosin is constrained by its long hydrophobic domain. J Biol Chem 2002, 277(10):8602-10.

25. Abell BM, Hahn M, Holbrook LA, Moloney MM: Membrane topology and sequence requirements for oil body targeting of oleosin. Plant J 2004, 37(4):46I-70.

26. Ostermeyer AG, Ramcharan LT, Zeng Y, Lublin DM, Brown DA Role of the hydrophobic domain in targeting caveolin-I to lipid droplets. J Cell Biol 2004, 164:69-78.

27. Subramanian V, Garcia A, Sekowski A, Brasaemle DL: Hydrophobic sequences target and anchor perilipin $A$ to lipid droplets. J Lipid Res 2004, 45(I I):1983-9I.

28. Targett-Adams P, Chambers D, Gledhill S, Hope RG, Coy JF, Girod A, McLauchlan J: Live cell analysis and targeting of the lipid droplet-binding adipocyte differentiation-related protein. Biol Chem 2003, 278(18): 15998-6007.

29. Kim HU, Wu SS, Ratnayake C, Huang AH: Brassica rapa has three genes that encode proteins associated with different neutral lipids in plastids of specific tissues. Plant Physiol 200I, | 26:330-4|.

30. Sembiosys [http://www.sembiosys.com/Index.aspx]

3I. Moloney M, Boothe J, Van Rooijen G: Oil bodies and associated proteins as affinity matrices. US Patent 65094532003.

32. Markley NA, Nykiforuk CL, Boothe JG, M MM: Producing Proteins Using Transgenic Oilbody-Oleosin Technology. BioPharm International 2006.

33. Nykiforuk CL, Boothe JG, Murray EW, Keon RG, Goren HJ, Markley NA, Moloney MM: Transgenic expression and recovery of biologically active recombinant human insulin from Arabidopsis thaliana seeds. Plant Biotechnology Journal 2006, 4:77-85.

34. Jolivet P, Roux E, D'Andrea S, Davanture M, Negroni L, Zivy M, Chardot T: Protein composition of oil bodies in Arabidopsis thaliana ecotype WS. Plant Physiol Biochem 2004, 42(6):50I-9.

35. Lichtenthaler HK: Plastoglobuli and the fine structure of plastids. Endeavor 1968, 27: | 44-| 49.

36. Vishnevetsky M, Ovadis M, Zuker A, Vainstein A: Molecular mechanisms underlying carotenogenesis in the chromoplast: multilevel regulation of carotenoid-associated genes. Plant J 1999 , 20(4):423-31.

37. Ghosh S, Mahoney SR, Penterman JN, Peirson D, Dumbroff EB: Ultrastructural and biochemical changes in chloroplasts during Brassica napus senescence. Plant Physiol Biochem 200I, 39:777-784.

38. Keskitalo J, Bergquist G, Gardestrom P, Jansson S: A cellular timetable of autumn senescence. Plant Physiol 2005, 139(4): 1635-48.

39. Yamada K, Lim J, Dale JM, Chen H, Shinn P, Palm CJ, Southwick AM, Wu HC, Kim C, Nguyen M, Pham P, Cheuk R, Karlin-Newmann G, Liu SX, Lam B, Sakano H, Wu T, Yu G, Miranda M, Quach HL, Tripp M, Chang CH, Lee JM, Toriumi M, Chan MM, Tang CC, Onodera CS, Deng JM, Akiyama K, Ansari Y, Arakawa T, Banh J, Banno F, Bowser L, Brooks S, Carninci P, Chao Q, Choy N, Enju A, Goldsmith AD, Gurjal M, Hansen NF, Hayashizaki Y, Johnson-Hopson C, Hsuan VW, lida K, Karnes M, Khan S, Koesema E, Ishida J, Jiang PX, Jones T, Kawai J, Kamiya A, Meyers C, Nakajima M, Narusaka M, Seki M, Sakurai T, Satou M, Tamse R, Vaysberg M, Wallender EK, Wong C, Yamamura Y, Yuan S, Shinozaki K, Davis RW, Theologis A, Ecker JR: Empirical analysis of transcriptional activity in the Arabidopsis genome. Science 2003, 302(5646):842-6. 
40. Bauer J, Chen K, Hiltbunner A, Wehrli E, Eugster M, Schnell D, Kessler $\mathrm{F}$ : The major protein import receptor of plastids is essential for chloroplast biogenesis. Nature 2000, 403(6766):203-7.

41. Jin JB, Kim YA, Kim SJ, Lee SH, Kim DH, Cheong GW, Hwang I: A new dynamin-like protein, ADL6, is involved in trafficking from the trans-Golgi network to the central vacuole in Arabidopsis. Plant Cell 200I, I3(7):15II-26.

42. Bechthold N, Ellis J, Pelletier G: in planta Agrobacterium-mediated gene transfer by infiltration of Arabidopsis thaliana plants. CR Acad Sci Ser III Sci Vie 1993, 3 I6: I I94-I 199.

43. Clough SJ, Bent AF: Floral dip: a simplified method for Agrobacterium-mediated transformation of Arabidopsis thaliana. Plant J 1998, I6(6):735-43.

44. Rensink WA, Pilon M, Weisbeek P: Domains of a transit sequence required for in vivo import in Arabidopsis chloroplasts. Plant Physiol 1998, 1 1 8(2):691-9.

45. Wessel D, Flügge UI: $\mathbf{A}$ method for the quantitative recovery of protein in dilute solution in the presence of detergents and lipids. Anal Biochem 1984, I38:141-143.

46. Hiltbrunner A, Bauer J, Vidi PA, Infanger S, Weibel P, Hohwy M, Kessler F: Targeting of an abundant cytosolic form of the protein import receptor atTocl59 to the outer chloroplast membrane. J Cell Biol 200I, I54(2):309-I6.

Publish with Bio Med Central and every scientist can read your work free of charge

"BioMed Central will be the most significant development for disseminating the results of biomedical research in our lifetime. "

Sir Paul Nurse, Cancer Research UK

Your research papers will be:

- available free of charge to the entire biomedical community

- peer reviewed and published immediately upon acceptance

- cited in PubMed and archived on PubMed Central

- yours - you keep the copyright

Submit your manuscript here:

http://www.biomedcentral.com/info/publishing_adv.asp
BioMedcentral 